\title{
OP13
}

\section{LUMINOUS INTENSITY COMPARISON BASED ON NEW STANDARD LAMPS WITH LED REFERENCE SPECTRUM \\ Thorsten Gerloff et al.}

DOI 10.25039/x46.2019.OP13

from

CIE x046:2019

Proceedings

of the

29th CIE SESSION

Washington D.C., USA, June 14 - 22, 2019

(DOI 10.25039/x46.2019)

The paper has been presented at the 29th CIE Session, Washington D.C., USA, June 14-22, 2019. It has not been peer-reviewed by CIE.

(C) CIE 2019

All rights reserved. Unless otherwise specified, no part of this publication may be reproduced or utilized in any form or by any means, electronic or mechanical, including photocopying and microfilm, without permission in writing from CIE Central Bureau at the address below. Any mention of organizations or products does not imply endorsement by the CIE.

This paper is made available open access for individual use. However, in all other cases all rights are reserved unless explicit permission is sought from and given by the CIE.

CIE Central Bureau

Babenbergerstrasse 9

A-1010 Vienna

Austria

Tel.: +4317143187

e-mail: ciecb@cie.co.at

www.cie.co.at 


\title{
LUMINOUS INTENSITY COMPARISON BASED ON NEW STANDARD LAMPS WITH LED REFERENCE SPECTRUM
}

Gerloff, T. ${ }^{1}$, Kallenbach, L. ${ }^{1}$, Ledig, J. ${ }^{1}$, Schrader, C. ${ }^{1}$, Sperling, A. ${ }^{1}$, Pulli, T. ${ }^{2}$, Askola, J. ${ }^{2}$, Šmid, M. ${ }^{3}$, Kliment, P. ${ }^{3}$, Pons, A. ${ }^{4}$, Ferrero, A. ${ }^{4}$, Gál, P. ${ }^{5}$, Brida, G. ${ }^{6}$, Blattner, P. ${ }^{7}$, Stuker, F. ${ }^{7}$, Schneider, M. ${ }^{8}$, Dönsberg, T. $^{9}$, and Poikonen, T. ${ }^{9}$

${ }^{1}$ PTB, Braunschweig, GERMANY, ${ }^{2}$ Aalto University, Espoo, FINLAND, ${ }^{3}$ ČMI, Prague, CZECH REPUBLIC, ${ }^{4}$ CSIC, Madrid, SPAIN, ${ }^{5}$ BFKH, Budapest, HUNGARY, 6 INRIM, Turin, ITALY, 7 METAS, Bern-Wabern, SWITZERLAND, ${ }^{8}$ OSRAM OS, Regensburg, GERMANY, ${ }^{9} \mathrm{VTT}$, Espoo, FINLAND

thorsten.gerloff@ptb.de

DOI $10.25039 / \times 46.2019 .0 P 13$

\begin{abstract}
Photometric calibrations are mainly based on the use of scientific grade incandescent standard lamps [CIE 2002]. Most of the measurement methods and reference spectra used in photometry were developed long before modern solid-state lighting (SSL) products were invented and introduced into the lighting market. In addition, phasing-out of incandescent lamps for lighting applications poses a metrological problem: all lamps and luminaires measured in practice differ completely in their behaviour with respect to spectral distribution, flicker and aging, as compared to incandescent lamps used in photometer calibrations.
\end{abstract}

The European research project "Future photometry based on solid-state lighting products" (EMPIR 15SIB07 PhotoLED) has investigated the fundamental requirements for photometry based on white light-emitting diode (LED) sources. The project partners have analysed many hundreds of LED spectra and derived suitable LED reference spectra for photometric calibrations.

In this article we present the practical advantages and disadvantages of luminous intensity standards based on white LEDs with very similar spectra to the hypothetical reference spectrum developed in the project. Furthermore, we discuss their suitability for metrological comparisons of the luminous intensity.

Keywords: Photometry, luminous intensity, metrological comparison, LED standard lamp, Standard illuminant A, LED reference spectrum

\section{Motivation}

For energy efficiency reasons, the large shift from incandescent to LED lamps for lighting applications has taken place almost everywhere in the world. Due to the conversion of production, the availability of high-quality standard incandescent lamps for metrological and scientific applications is no longer guaranteed.

On the metrological side, the problem arises here that on the one hand the calibration of photometers for general use has so far taken place by means of incandescent lamps with a high similarity to standardised Illuminant $A$, on the other hand, however, predominantly LEDbased lamps are measured.

Due to the large spectral differences between the two lamp types, the spectral mismatch of the $V(\lambda)$-filters used in photometers can have a much more significant effect than when measuring incandescent lamps [ISO/CIE 2014]. An approach examined in the European EMPIR project "15SIB07 PhotoLED" is to define a new LED reference spectrum and to realize calibration light sources with this spectrum. By calibrating the photometers against these sources, a reduction in the measurement deviations due to the lower spectral mismatch is to be expected. 


\section{Proposed LED Reference Spectrum}

One project goal was the determination of an LED reference spectrum for the calibration of photometers, which is representative of as many LED light sources on the market as possible. For this purpose, the spectral distributions of 1516 LED products and the spectral responsivities of 107 photometers of various types were measured. The LED spectra were divided into groups of different similar correlated colour temperature (CCT) and a representative spectrum was mathematically calculated for each group (appr. $2730 \mathrm{~K}$, appr. $3000 \mathrm{~K}$, appr. $4100 \mathrm{~K}$, appr. $5100 \mathrm{~K}$ and appr. $6600 \mathrm{~K}$ ). This approach avoids the preference for manufacturer-specific LEDs and is intended to increase the acceptance as independent but representative LED-spectrum.

The spectral mismatch errors were simulated for all combinations of these five hypothetical reference calibration spectra for these 107 photometers with the lamp spectra of all LEDs. The results of the simulation were weighted according to various criteria (e.g. field of application).

Data analysis revealed a minimum of the averaged spectral mismatch error for LED measurements if the hypothetical spectrum with a CCT of $4100 \mathrm{~K}$ is used for calibration of photometers instead of standard illuminant A or one of the other 4 LED spectra.

Therefore, the LED spectrum with a CCT of $4100 \mathrm{~K}$ was chosen as base spectrum of the light sources presented here. Project partners selected suitable LEDs from production for the construction of the luminous intensity standard sources.

Further information on the development of the white LED illuminants and the chosen LED reference spectrum can be found in the literature [Kokka 2018]. Additionally, these new illuminants for different LED types are introduced in the $4^{\text {th }}$ edition of CIE publication on Colorimetry [CIE 2018].

\section{Design of the luminous intensity standard lamp}

The design of the reference luminous intensity source type A (LIS-A) is based on the dimensions and typical luminous intensity value of the widespread standard lamps OSRAM WI 41 / G. The housing consists of an aluminum cylinder with a diameter of $100 \mathrm{~mm}$ and a length of $70 \mathrm{~mm}$. The main light output direction is horizontal (Fig. 1). A reference mark on the housing and a jig (not shown) allows a highly reproducible alignment of the position and orientation. For achieving an upright position, a level is integrated to the housing.

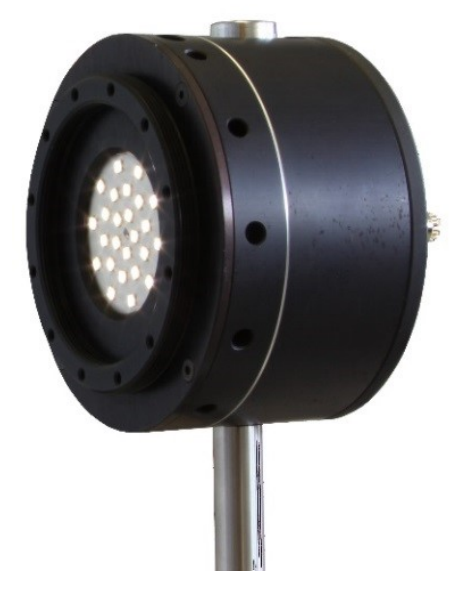

Figure 1 - Luminous intensity source type A (LIS-A).

In order to achieve a substantial insensitivity to ambient temperature and air flows, the heat exchange takes place via an internal heat sink, which is actively cooled by a fan on the back. The air outlet can be switched between front and side (Fig. 2). The LED-chip temperature is regulated by means of an underlying Peltier element via an integrated active temperature controller. 


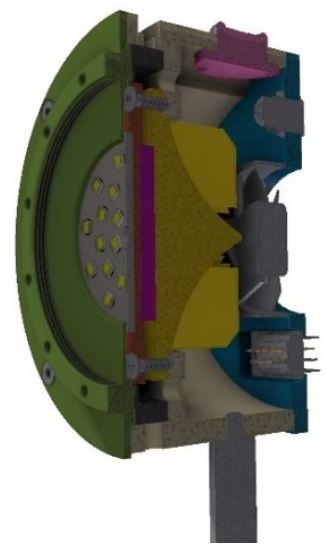

Figure 2 - CAD-model of LIS-A.

On the printed circuit board (PCB) with a metal core 25 SMD-LEDs are arranged in three concentric rings (with 5, 10 and 10 LEDs and corresponding diameters of appr. 13, 25 and $7 \mathrm{~mm}$ ) and connected to 5 strings with 5 LEDs each. This enables to vary the effective diameter of the light source by separate activation or to improve the homogeneity over the module surface. The luminous intensity is about $245 \mathrm{~cd}$ in the standard configuration for $4100 \mathrm{~K}$. A group of 10 artefacts was manufactured in the frame of the PhotoLED-project (Fig. 3).

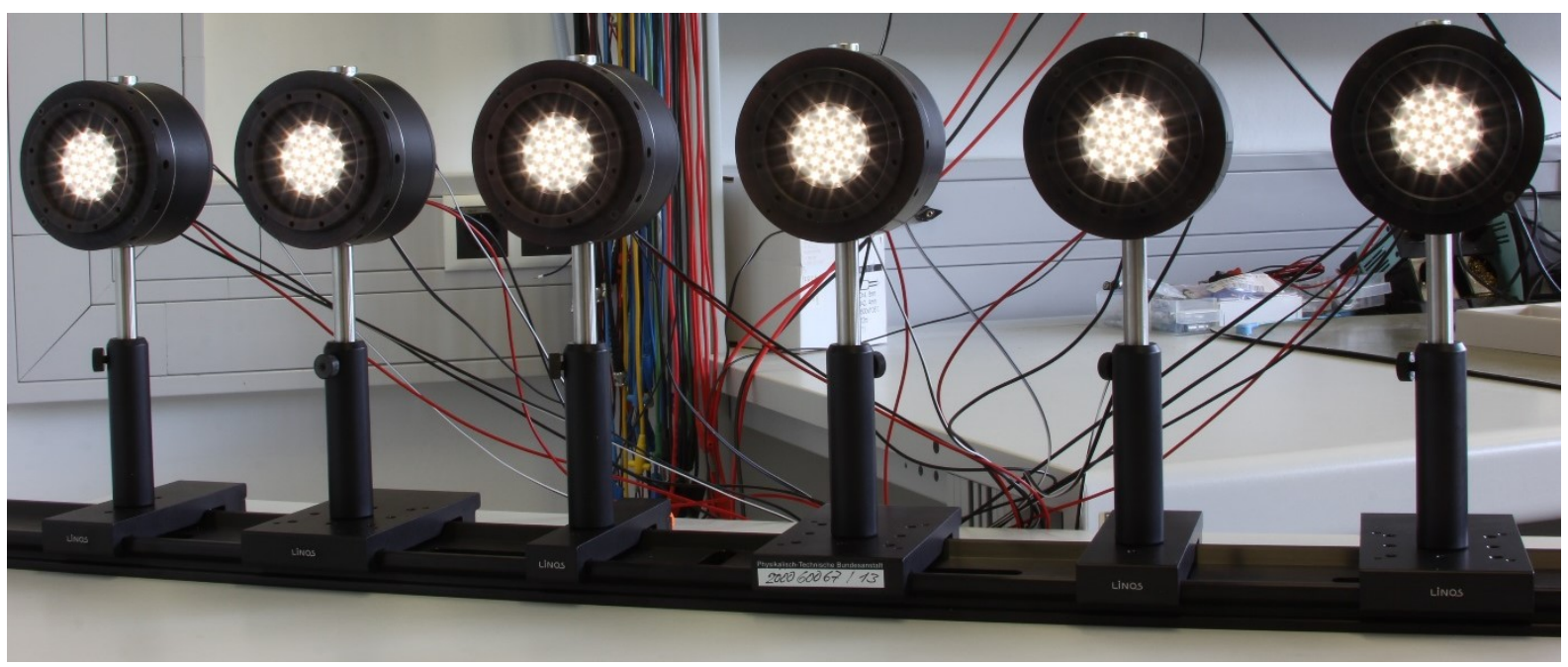

Figure 3 - 6 LIS-A artefacts equipped with appr. $4100 \mathrm{~K}$ LEDs.

\section{Luminous Intensity comparison}

The aim of the comparison is to assess the quality and robustness of the LED illuminance and luminous intensity standard LIS-A developed in the PhotoLED project, and to study the measurement uncertainties reached by using traditional photometers with $V(\lambda)$, unfiltered radiometers based on predictable quantum-efficient detectors (PQEDs) as well as trap detectors constructed using commercial photodiodes.

A total of 8 LIS-A artefacts were designed, constructed and characterized at PTB. Groups of 2 LIS-As each were formed and sent to the partners for the comparison measurements. Several European NMIs will have completed LIS-A calibrations for the physical quantities of luminous intensity and illuminance by the end of the project in August 2019. The first and last calibrations within the comparison are performed for each artefact group at PTB.

Since the comparison is still ongoing, we cannot yet publish all results. However, we would like to discuss some aspects relevant to the calibration of the LED standards presented. 


\subsection{Artefacts}

The LIS-A artefacts for the comparison are white-LED-based standard lamps with a CCT of about $4020 \mathrm{~K}$ and a luminous intensity of about $245 \mathrm{~cd}$. The lamps are operated using an external DC current of approximately $65 \mathrm{~mA}$ with a supply voltage of about $75 \mathrm{~V}$.

The internal temperature control unit keeps the LEDs PCB surface at the temperature of about $45^{\circ} \mathrm{C}$. All lamps were seasoned for at least 1000 hours at PTB to reduce aging effects and to ensure stability during the comparison. The relative changes of the photocurrent of a monitor photometer for the last 360 hours of seasoning are shown in Fig. 4. The mean change in the light intensity of the modules is significantly lower than $0.1 \%$ for the last 100 hours of seasoning.

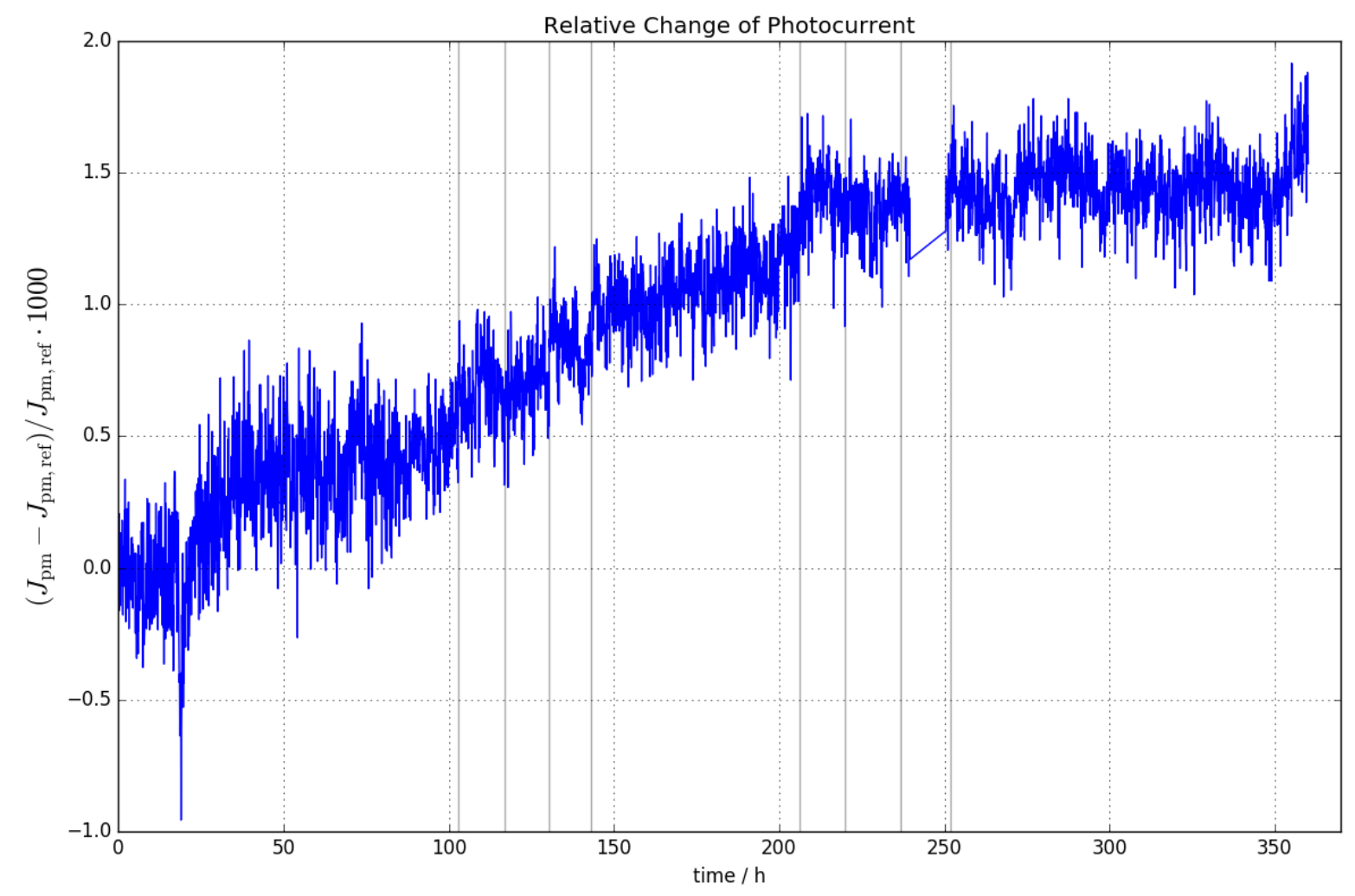

Figure 4 - Relative change of the photocurrent of a monitor photometer during seasoning.

\subsection{Mounting and alignment}

Plots in Fig. 5 show the relative change in luminous intensity as a function of the angle at which the light source was rotated along its horizontal and vertical axes of rotation. This was measured with four photometers of different types. Due to the symmetrical structure and the lack of reflection surfaces, a uniform and symmetrical curve close to the theoretically expected cosine for a Lambertian source is found. 

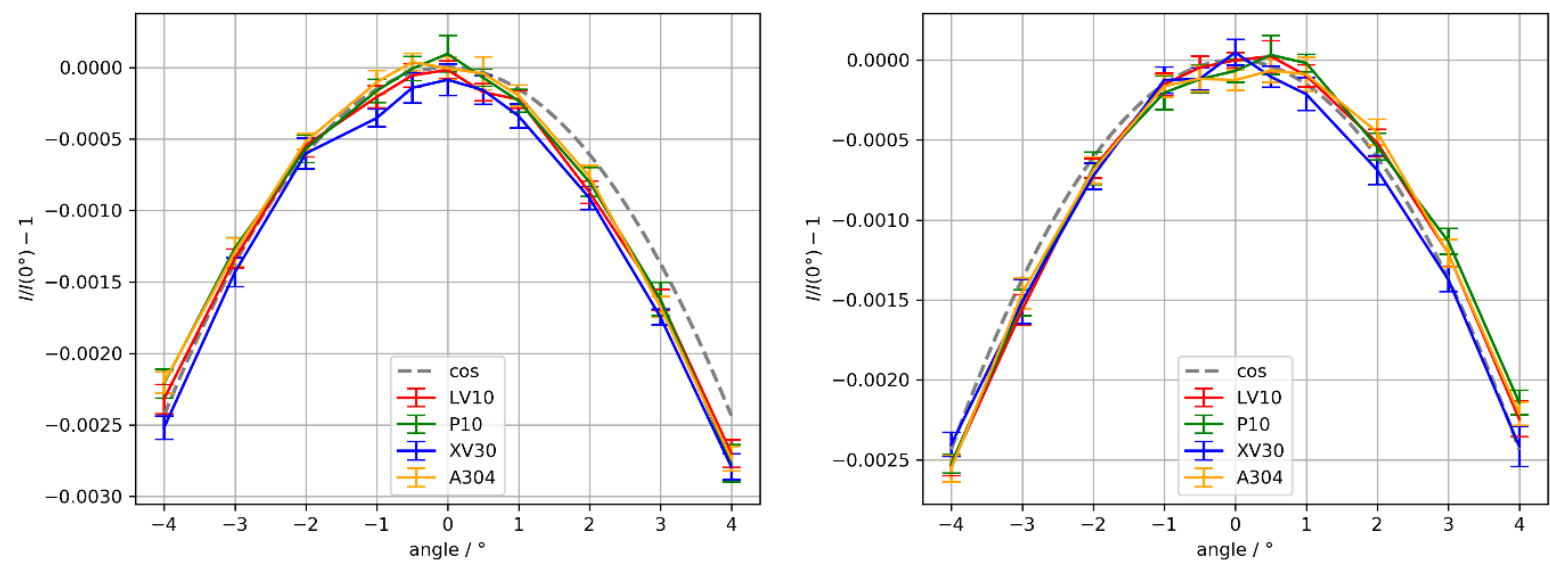

Figure 5 - Relative changes of luminous intensity for different angular alignments. The left image shows rotation around the horizontal axis. The right image shows rotation around the vertical axis.

\subsection{Ambient temperature}

Figure 6 shows the relative changes in illuminance with temperature with respect to the value at the nominal temperature $25^{\circ} \mathrm{C}$ for 6 modules. The influence of the ambient temperature and humidity was determined in a climate chamber with an external photometer. The ambient temperature was varied between $20^{\circ} \mathrm{C}$ and $30^{\circ} \mathrm{C}$ and each measurement was done after 20 minutes stabilization time. All measurements were carried out at $50 \%$ relative humidity.

The deviations are below $1.5 \cdot 10^{-4}$, which can be regarded as negligible especially considering the standard deviations at each measuring point.

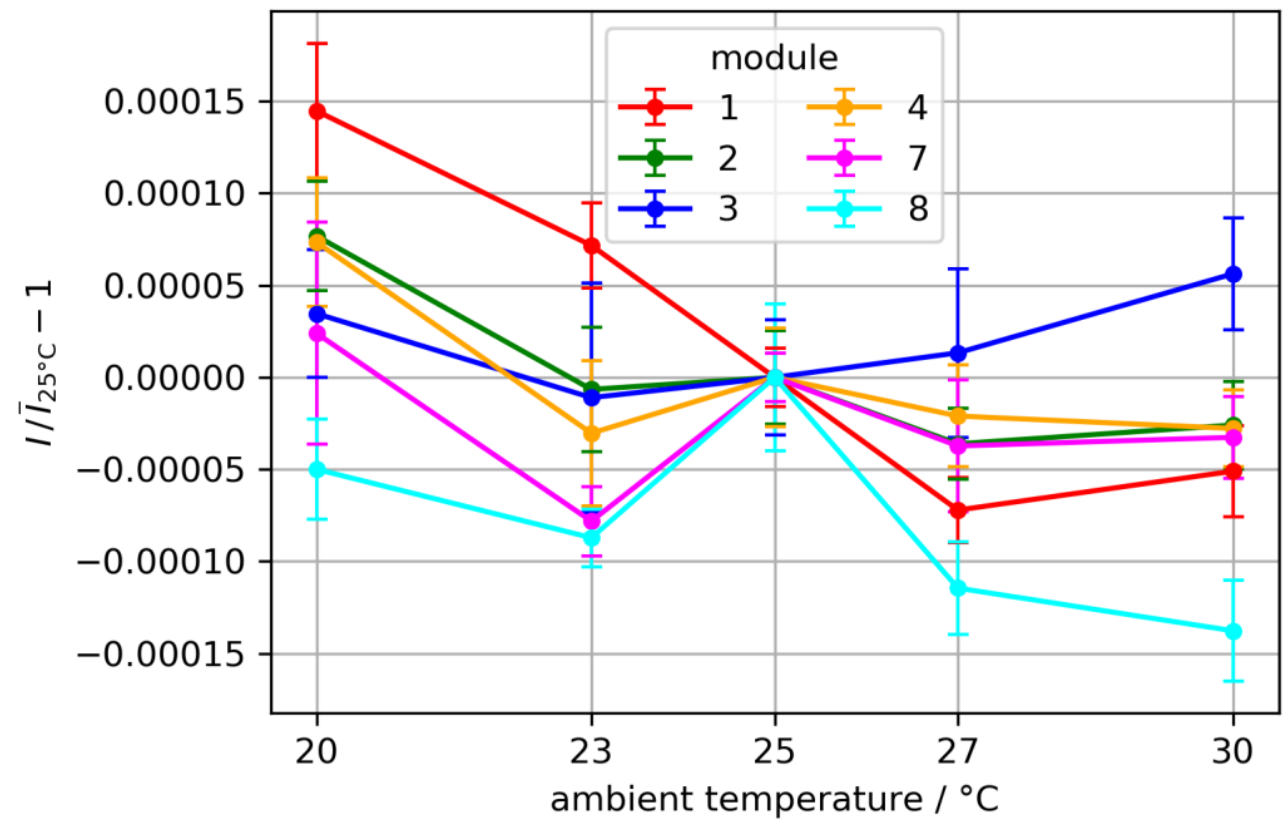

Figure 6 - Relative changes of illuminance for different ambient temperatures.

\subsection{Repeatability}

In order to obtain information about the current state of seasoning and the repeatability of the operating point, the modules were operated cyclically. One cycle includes 30 minutes in the onstate, followed by a 15-minute cool down phase in the off-state. During the on-state phase, a measuring point was recorded every 2 minutes. For each on-state phase, a stable operating 
point was reached after a burn-in period of 8-10 minutes. The last 20 minutes of each on-state cycle for a sufficiently seasoned artefact are exemplary shown in Figure 7 . The upper diagram shows the relative changes of lamp current and lamp voltage.

The middle diagram shows relative changes in the photocurrent of the photometer. In this case yellow is the relative change of the measured value, and orange the relative change for the value corrected to nominal lamp current.

The lower part shows the respective on and off times and the ambient temperature (black curve). The module shows only minimal aging effects in these approximately 65 hours and a stability that is in the range of system noise.

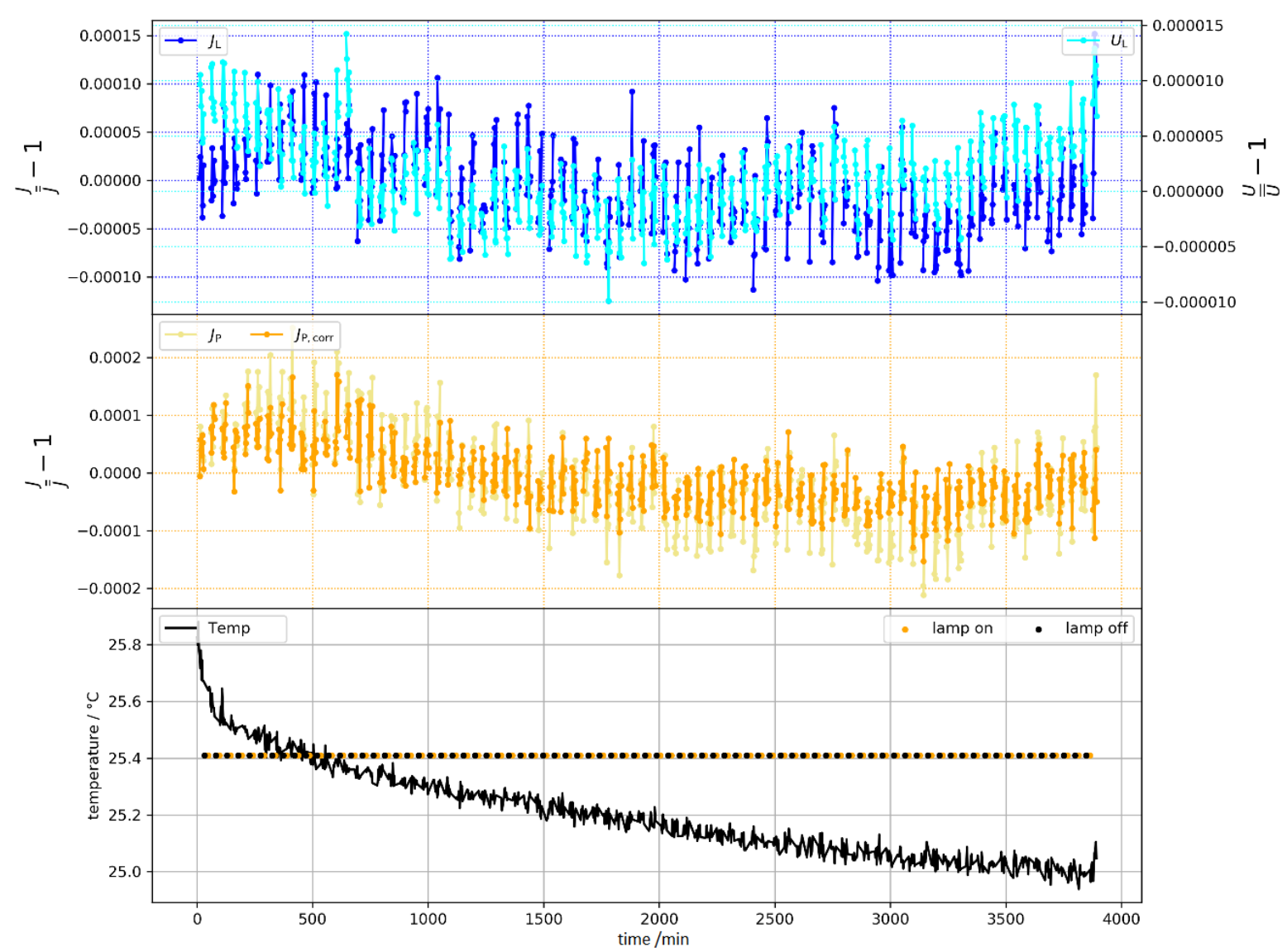

Figure 7 - Exemplary repeatability of one artefact.

\subsection{Spectral measurements}

The calibration of LED standards at NMI level requires precise knowledge of the spectral distribution. This allows to correct the spectral mismatch of the photometer. The quality of the spectral measurement has a significant influence on the resulting measurement uncertainty of the luminous intensity calibration. 

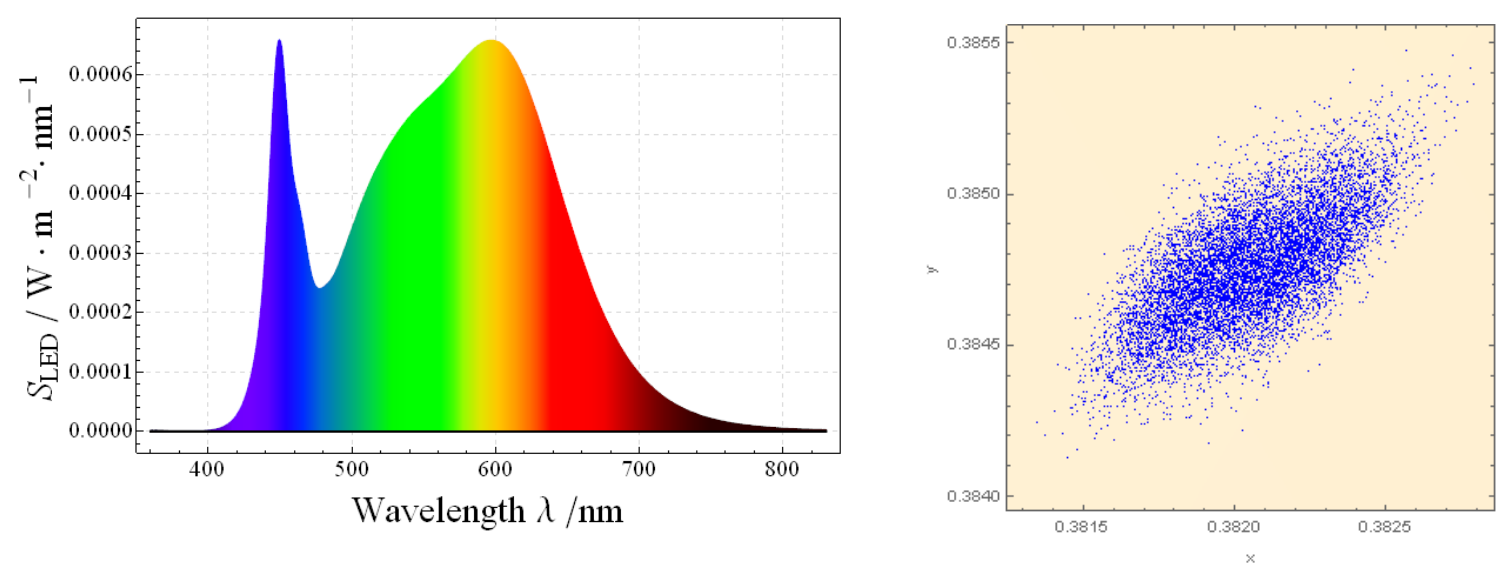

Figure 8 - Left: Spectral distribution of one artefact. Right: Result of 10000 Monte Carlo
simulations of the chromaticity.

Monte Carlo (MC) simulations were performed for the analysis of the spectral data for each LISA. Exemplary results of the simulations for chromaticity and spectral mismatch factor for one artefact are summarised in Table 1. The simulations are based on simulated wavelength shift, wavelength noise of each spectrometer pixel, many measurements of the spectral irradiance of the artefacts, spectral irradiance of FEL standard lamps for spectroradiometer calibration, the spectral sensitivity of the photometer and other datasets. The result of 10000 simulations of the chromaticity is shown in fig. 8.

\section{Table 1 - Results of spectral measurements}

\begin{tabular}{|c|c|c|c|}
\hline \multirow{2}{*}{\multicolumn{2}{|c|}{ Chromaticity $(\mathrm{x}, \mathrm{y})$ : }} & $\mathrm{x}$ & $\mathrm{y}$ \\
\hline & & 0.38205 & 0.38475 \\
\hline & SMU Chromaticity & 0.00023 & 0.00019 \\
\hline Exp.MU Chromaticity & (Multivariate GAUSS-Distribution $\rightarrow \mathrm{k}=2.45$ (refer GUM S2 6.5 .4 table 1)) & 0.00057 & 0.00046 \\
\hline \multicolumn{2}{|r|}{ Correlations } & $\left(\begin{array}{cc}1 . & 0.684879 \\
0.684879 & 1 .\end{array}\right.$ & \\
\hline & Spectral Mismatch Factor & 1.0009 & \\
\hline & SMU Spectral Mismatch Factor & 0.0014 & \\
\hline & Number of MC Simulations & 10000 & \\
\hline & Wavelength Noise $/ \mathrm{nm}$ & \multirow{2}{*}{$\begin{array}{l}0.2 \\
0.2\end{array}$} & \\
\hline & Wavelength Shift $/ \mathrm{nm}$ & & \\
\hline
\end{tabular}

\subsection{Measurement uncertainty of luminous intensity}

Uncertainties are determined from up to 30 individual contributions that are influenced by the operation parameters, measurement setup and environmental conditions (orientation of the LISA, thermal conditions, air movement in the laboratory, digital voltmeter, etc.). The specific characteristics of the measuring instruments and their effects to the luminous intensity calibration are considered in detail.

For the sake of clarity, some uncertainty contributions that have neither a significant impact nor a particular relevance are omitted in the following discussion.

The specified uncertainties of the contributions are exemplary of typical calibrations of standard LEDs with similar properties. No particular effort has been made to minimize the uncertainties to show representative results for application as a transfer standard.

The measurement uncertainty contributions are listed and sorted into uncertainty budgets. The components are treated as uncorrelated.

The used model to determine the luminous intensity $I_{\mathrm{LED}}$ is:

ILED $=C_{\text {Spectral }} C_{\text {StrayLight }} C_{\text {Alignment }} C_{\text {AmbTemp }} \operatorname{distance}\left(\frac{J_{\text {LED }}}{J_{\text {LED } 0}}\right)^{-\alpha}\left(\frac{U_{\text {LED }}}{U_{\text {LED } 0}}\right)^{-\beta} \frac{f_{\text {AmpLight }}-f_{\text {AmpDark }}}{W_{f} R_{g} S_{V}}$

where the symbols are given in the following table, together with the relevant standard uncertainty contributions: 


\begin{tabular}{|c|c|c|c|c|c|c|c|c|}
\hline DESCRIPTION & UNIT & SYMBOL & VALUE & $\frac{\text { UNCERTAINTY }}{(\underline{\text { IN })}}$ & DOF & SENSITIVITY & UNCERTAINTY & $\frac{\text { REL. }}{\text { UNCERTAINTY }}$ \\
\hline LED Nominal Current & A & $J_{\text {LED } 0}$ & 0.065 & 0 & $\infty$ & 2779.39 & 0. & 0. \\
\hline LED Nominal Voltage & $\mathrm{V}$ & $\mathrm{U}_{\mathrm{LED} 0}$ & 70.653 & 0 & $\infty$ & 10.2626 & 0 . & 0 . \\
\hline Exponent LED Voltage Corr. & 1 & $\beta$ & 2.97 & 0.09 & 19 & 0.00314445 & 0.000283 & $1.15919 \times 10^{-6}$ \\
\hline Photo Current Amplifier Dark & $\mathrm{Hz}$ & $\mathrm{f}_{\text {AmpDark }}$ & 1574.02 & 3.14804 & 49 & -0.00150284 & -0.00473101 & -0.0000193786 \\
\hline Exponent LED Current Corr. & 1 & $\alpha$ & 0.74 & 0.05 & 19 & -0.172363 & -0.00861813 & -0.0000353006 \\
\hline Corr. Ambient Temperature & 1 & $\mathrm{C}_{\text {AmbTemp }}$ & 1 & 0.00005 & 19 & 244.135 & 0.0122068 & 0.00005 \\
\hline LED Current & A & $J_{\text {LED }}$ & 0.0650459 & $6.50459 \times 10^{-6}$ & 49 & -2777.43 & -0.018066 & -0.000074 \\
\hline Amplification Photo Current Amplifier & $\Omega$ & $\mathrm{Rg}_{\mathrm{g}}$ & $2.99954 \times 10^{6}$ & 299.954 & 99 & -0.0000813909 & -0.0244135 & -0.0001 \\
\hline Photo Current Amplifier & $\mathrm{Hz}$ & $\mathrm{f}_{\text {AmpLight }}$ & 164023 & 32.8046 & 49 & 0.00150284 & 0.0493002 & 0.000201938 \\
\hline LED Voltage & $\mathrm{V}$ & $\mathrm{U}_{\mathrm{LED}}$ & 70.6521 & 0.00706521 & 49 & -10.2627 & -0.0725082 & -0.000297 \\
\hline UF/converter conversion factor & $\frac{\mathrm{Hz}}{\mathrm{V}}$ & $w_{f}$ & 50078.3 & 15.0235 & 49 & -0.00487507 & -0.0732406 & -0.0003 \\
\hline Corr. Factor for Straylight & 1 & CStraytight & 0.9995 & 0.0005 & 39 & 244.257 & 0.122129 & 0.00050025 \\
\hline Distance & $\mathrm{m}$ & distance & 2.50001 & 0.001 & 49 & 195.307 & 0.195307 & 0.000799997 \\
\hline Corr. f. LED Alignment & 1 & $\mathrm{C}_{\mathrm{Al} \text { ignment }}$ & 1 & 0.0008 & 19 & 244.135 & 0.195308 & 0.0008 \\
\hline Corr. Factor f. Spec. Mismatch Photom. & 1 & $\mathrm{C}_{\text {Spectra1 }}$ & 1.0009 & 0.00137 & 49 & 243.916 & 0.334165 & 0.00136877 \\
\hline Luminous Responsivity Photometer & $\frac{\mathrm{A}}{1 \mathrm{x}}$ & $s_{\mathrm{v}}$ & $2.7684 \times 10^{-8}$ & $6.921 \times 10^{-11}$ & $\infty$ & $-8.81864 \times 10^{9}$ & -0.610338 & -0.0025 \\
\hline Result: Luminous Intensity & cd & $I_{\text {LED }}$ & 244.135 & - & 945 & - & 0.767857 & 0.00314521 \\
\hline
\end{tabular}

This leads to a relative expanded uncertainty of $0.64 \%$ for the luminous intensity calibration of one LIS-A artefact:

\begin{tabular}{|c|c|c|c|c|c|c|c|}
\hline DESCRIPTION & UNIT & SYMBOL & VALUE & $k$ & $\begin{array}{c}\text { CONFIDENCELEVEL } \\
\text { EXPAND. } \\
\text { UNCERTAINTY }\end{array}$ & $\begin{array}{c}\text { REL. EXPAND. } \\
\text { UNCERTAINTY }\end{array}$ \\
\hline Result: Luminous Intensity & cd & I $_{\text {LED }}$ & 244.135 & 2.025 & 0.9545 & 1.55491 & 0.00636905 \\
\hline
\end{tabular}

\subsection{Results of the comparison}

At the time of writing this paper, the comparison is still ongoing. Therefore, we cannot publish the results of the already completed measurements. We are looking forward to present the preliminary results of the luminous intensity comparison in the oral presentation.

\section{Acknowledgements}

The work leading to this study is partly funded by the European Metrology Programme for Innovation and Research (EMPIR) Project 15SIB07 PhotoLED "Future Photometry Based on Solid State Lighting Products". The EMPIR initiative is co-funded by the European Union's Horizon 2020 research and innovation programme and the EMPIR Participating States.

The authors thank Stefan Pendsa (PTB) and Mario Taddeo (PTB) for the electrical and mechanical development and construction of the LIS-A artefacts.

\section{References}

CIE 2002. CIE 149:2002. The use of tungsten filament lamps as secondary standard sources. Vienna: CIE.

CIE 2018. CIE 015:2018. Colorimetry, 4th Edition. Vienna: CIE.

ISO/CIE 2014. Characterization of the Performance of Illuminance Meters and Luminance Meters, ISO/CIE Standard 19476:2014(E). Vienna: CIE

KOKKA, A. 2018, Development of white LED illuminants for colorimetry and recommendation of white $L E D$ reference spectrum for photometry. Metrologia. 55. 526-534 